\title{
Retraction: Fluorobodies combine GFP fluorescence with the binding characteristics of antibodies
}

\author{
Ahmet Zeytun, Andreas Jeromin, Bethe A Scalettar, Geoffrey S Waldo \& Andrew RM Bradbury \\ Nat. Biotechnol. 21, 1473-1479 (2003)
}

In this article, we concluded that inserting HCDR3 sequences derived from antibodies into a particular stable form of GFP created intrinsically fluorescent affinity reagents, which we termed 'fluorobodies.' We have recently realized that the strategy used to generate fluorobodies was flawed: the adaptor sequences reported contained additional nucleotides that introduced translational frameshifts at each HCDR3 insertion site. We expect this to have resulted in the creation of tripartite nonfluorescent "GFP-HCDR3 fragments" with the following structures: N-terminal in-frame GFP peptide/HCDR3 sequence/out-of-frame C-terminal GFP peptide.

Reexamination of the primary bacterial stocks of fluorobodies has revealed that these were not monoclonal, as originally assumed, but contained plasmid mixtures encoding both unmodified GFP and GFP-HCDR3 fragments. Segregation of these plasmids revealed that only colonies containing unmodified GFP genes were green.

Following these discoveries, we have subsequently carried out immunofluorescence experiments with the remaining anti-tubulin fluorobody used in the published paper and obtained identical (microtubule-like) staining patterns, whereas GFP or anti-tubulin fluorobody prepared from frozen bacterial stocks gave nonspecific staining. With this exception, no original fluorobody protein preparations were available, and attempts to reproduce other results reported in the paper with fluorobodies expressed from frozen bacterial stocks were unsuccessful. We have reason to believe these stocks may have been compromised, and that the similarity of these stored stocks to the original stocks is questionable.

To determine whether correctly assembled fluorobodies would be functional, we have subsequently generated libraries of HCDR3s inserted at the single loop sites described in the paper, as well as into three (1-3) and four (1-4) loops simultaneously, within the context of a phage display vector. Only one of the single loop libraries (loop 3: in which $30 \%$ of clones had fluorescence greater than $5 \%$ of the GFP fluorescence, with a maximum of $12 \%$ ) contained significant numbers of fluorescent clones; the rest were essentially nonfluorescent.

With the exception of the immunofluorescence data (produced by A.J. and B.A.S. using material prepared by A.Z.), all experiments were carried out by A.Z. in A.R.M.B.'s laboratory. We are presently unable to explain the biological activity described in the original publication. The possibility that this arose from noncovalent association of GFP with GFP-HCDR3 fragments in bacteria containing multiple plasmids is presently under investigation. In light of these findings, all authors agree that this study has not demonstrated the creation of fluorobodies as described, and consequently wish to unanimously retract the paper. 